\title{
Aspectos clínico-epidemiológicos del aborto en un hospital de Upata. Estado Bolívar-Venezuela
}

Erly Pérez-Arciniegas ${ }^{1,3}$, Daviana Godoy-Albornoz ${ }^{1,7}$, Deymar Quiroz-Figuera ${ }^{1,4}$, Deynalia QuirozFiguera ${ }^{1,4}$, Carlos Tovar-Thomas ${ }^{1,6}$, Andrea Romero-Herrera ${ }^{1,3}$, Samuel Yary-Maestracci ${ }^{1,6}$, Jacqueline Brown-Davis ${ }^{1,4}$, Arianna Alzolay-Belisario ${ }^{1,5}$, Jorge Yzhac-Silva ${ }^{1,8}$, Nafxiel Brito- Nuñez ${ }^{2,3}$

${ }^{1}$ Hospital "Gervasio Vera Custodio", Upata. Estado Bolívar, Venezuela. ${ }^{2}$ Departamento de Morfología. Universidad de Oriente. Núcleo Bolívar. Venezuela. ${ }^{3}$ Hospital “Tulio López Ramírez”. Barrancas del Orinoco. Estado Monagas. Venezuela. ${ }^{4}$ Hospital Dr. "Luis Razetti”. Tucupita. Estado Delta Amacuro. Venezuela. ${ }^{5}$ Hospital tipo 1 de Uracoa. Estado Monagas. Venezuela. ${ }^{6}$ Ambulatorio rural tipo 1 "La ceiba". Estado Bolívar. Venezuela. ${ }^{7}$ Ambulatorio Urbano tipo II "Carmen Narcisa Iradi”. Guasipati. Estado Bolívar. Venezuela. ${ }^{8}$ Ambulatorio rural tipo II "El Pao". Estado Bolívar. Venezuela.

\section{RESUMEN}

Introducción. El aborto se ha convertido en un problema de salud pública. Una las causas que podría estar influyendo para su aumento es la falta de información sobre el control prenatal, por lo anterior, este es un tema que no escapa de la realidad y de la práctica médica.

Objetivo. Caracterizar clínica y epidemiológicamente el aborto.

Materiales y Métodos. Estudio observacional, descriptivo, retrospectivo, realizado en el Hospital "Gervasio Vera Custodio" UpataEstado Bolívar, Venezuela, durantel periodo comprendido de enero a diciembre de 2013. Se recolecto datos de 207 historias clínicas de mujeres con diagnóstico de aborto. En el análisis estadístico se aplicó la prueba chi ${ }^{2}$ de Pearson.

Resultados. Se encontró que la edad prevalente de aborto fue de 21 a 30 años con $42 \%$. La edad gestacional más frecuente de las pacientes fue de 1 a 8 semanas con $50 \%$. De estas $93,5 \%$ no cumplieron control prenatal. El tipo de aborto más frecuente fue espontáneo con $98,5 \%$. Se realizó legrado uterino a 97\%, de las cuales, 8,5\% presentaron complicaciones. Se encontraron diferencias estadísticamente significativas al relacionar la edad materna con la edad gestacional. Conclusiones. Existió alta prevalencia de aborto espontáneo en el grupo de 21 a 30 años de edad, presentados entre las semanas 1 a 8 de gestación. La técnica más segura fue el legrado uterino. Finalmente, se encontró bajo cumplimiento del control prenatal.

Palabras Clave: Aborto, control prenatal, edad gestacional

\section{ABSTRACT \\ Clinical and epidemiological aspects of abortion in a hospital of Upata. State Bolivar Venezuela \\ Introduction. The abortion has become a public health problem may be due to the lack of information about prenatal care, so that this is an issue which does not escape reality and everyday medical practice. \\ Objective. Characterize the abortion clinic and epidemiologically. \\ Materials and Methods. An observational, descriptive, retrospective study at the Hospital}

Autor para correspondencia: Erly Pérez Arciniegas, Urbanización Vista Hermosa I, vereda 2, casa \# 27, Upata. Estado Bolívar, Venezuela E-mail:erlyp.ar88@gmail.com

Recibido: el 18 de mayo de 2015 Aceptado para publicación: el 10 de agosto de 2015

Este documento está disponible en http://www.revbiomed.uady.mx/pdf/rb162712.pdf

Vol. 27, No. 1, enero-abril de 2016 


\section{Pérez-Arciniegas et al}

"Gervasio Vera Custodio" Upata-Ciudad Bolivar, Venezuela was performed from January to December 2013. Data from 207 women with diagnosed a diagnosis of abortion were collected from medical records. Pearson chi-square test was applied for the statistical analysis.

Results. It was found that abortion was prevalent among 21-30 years old with a 42\%. The most common gestational age of abortion was 1 to 8 weeks in the $50 \%$ and $93.5 \%$ of them did not meet prenatal care. The most frequent abortion was spontaneous in $98.5 \%$ ot the patients. Curettage was performed in $97 \%$, of which $8.5 \%$ had complications. Statistically significant differences were found to maternal age related to gestational age.

Conclusion. There was high prevalence of abortion in the group of 21-30 years of age with 1 to 8 weeks of gestation. The safest practice was the uterine curettage. Finally, the mayority of the cases had no prenatal care.

Key words: abortion, birth control, gestational age

\section{INTRODUCCIÓN}

El aborto, del lat. Abortus, según la Real Academia Española, es la interrupción del embarazo por causas naturales o deliberadamente provocadas que, puede constituir eventualmente un delito (1). Según la OMS, el aborto es la expulsión o extracción uterina de un embrión o de un feto de menos de 500 gramos, lo que corresponde con una edad gestacional de 20 a 22 semanas (2).

Mundialmente, ocurren 210 millones de embarazos anuales de los cuales 80 millones son no planificados (3). En Venezuela, de acuerdo al anuario de morbilidad del Ministerio del Poder Popular para la Salud, en el estado Bolívar la tasa de consultas a los centros de salud por emergencias de abortos es de 380,9 constituyendo $0,2 \%$ del total de las consultas, lo que a su vez representa una tasa especifica de 1,0 por cada 100.000 mujeres entre 15 y 49 años de edad $(4,5)$.

El aborto presenta diferentes clasificaciones: Según el tiempo de gestación se denomina aborto temprano al que ocurre antes de la $12^{\mathrm{a}}$ semana de gestación y aborto tardío al que se presenta entre las 12 y las 20 semanas de gestación(6,7). El 80-85\% de los abortos espontáneos pertenecen al primer grupo (8).

Otra posible clasificación es aquella que contempla el origen del aborto; esta incluye el aborto inducido, provocado o voluntario, en el que se usa maniobras destinadas a interrumpir el embarazo y el aborto espontáneo o involuntario, en el cual no interviene la voluntad de la madre ni de terceros $(9,10)$. Según su evolución, se presenta la amenaza de aborto, aborto inminente o inevitable que presenta ciertas circunstancias obstétricas que condicionan la irreversibilidad del proceso y el aborto diferido o retenido, que representa la muerte del embrión o feto sin su expulsión (7,9,11-13). Si el aborto se complica con una infección adquiere el calificativo de aborto séptico (14).

Según su terminación, puede existir el aborto completo cuando se expulsa la totalidad del producto de la concepción: el feto y sus anexos y el aborto en el que la expulsión es incompleta o parcial (8). De acuerdo a su frecuencia, se describe el aborto recurrente cuando ocurren dos o más abortos espontáneos consecutivos y aborto habitual, tres o más abortos $(6,7,9,15,16)$.

Otras causas se relacionan con factores genéticos, como los cromosopatías, endócrinos y ambientales. En los anatómicos se describen malformaciones congénitas y adquiridas como las anomalías mullerianas que son las más asociadas a perdida fetal, siendo el útero septo la más frecuente y de peor pronóstico en lo que se refiere a la reproducción. Entre los factores endócrinos se encuentran la hipersecreción de hormona luteinizante (LH), el hipotiroidismo, el hipertiroidismo, el hiperandrogenismo, la

\section{Revista Biomédica}


Epidemiología clínica del aborto en Venezuela

hiperprolactinemia, el síndrome metabólico, la resistencia a la insulina, el síndrome de ovario poliquístico, infecciosos y factores inmunológicos (6,7,16-18).

Los factores ambientales relacionados al consumo de café, cigarrillo, alcohol, deficiencia de ácido fólico, deficiencia de selenio, enfermedad celiaca, estrés, entre otros, también representan causas de aborto $(7,16,17)$.

Según la OMS, la aspiración manual endouterina (AMEU) es el método recomendado de evacuación endouterina. Existen otros métodos de evacuación endouterina como el legrado uterino instrumental; sin embargo, en el aborto temprano se están utilizando tratamientos farmacológicos como la administración de mifepristone en conjunto con misoprostol, reportando hasta $96 \%$ de efectividad en embarazos menores de 49 días de gestación $(8,19,20)$.

Entre las causas más importantes de muerte materna en los países Latinoamericanos y del Caribe se encuentran las complicaciones derivadas del aborto que representa $13 \%$ de la mortalidad materna global y $24 \%$, en la región de América Latina. Estas pueden ser inmediatas, mediatas o tardías. Las inmediatas se dividen en mayores y menores; dentro de las mayores se observan: muerte, perforaciones uterinas, desgarros cervicales, enfermedad pélvica inflamatoria, embolia de líquido amniótico, coagulación intravascular diseminada, infección y las menores son: dolor, adherencias cérvicouterinas, fiebre menor de 24 horas, hemorragia que no precisa trasfusión, aborto fracasado, endometritis, incontinencia urinaria. A largo plazo, se presenta muerte, cáncer de mama, cáncer de cérvix y la probabilidad de desarrollar en embarazos posteriores patologías como placenta previa, abortos espontáneos, embarazo ectópico y esterilidad (21-24).

La importancia que tiene el estudio del aborto se fundamenta en el hecho de que el mismo puede ocasionar problemas orgánicos, psicológicos y hasta la muerte. El aborto en las últimas décadas se ha convertido en un problema de salud pública, realidad a la cual no escapa la población de Upata, por ello se tiene como fin de determinar los aspectos epidemiológicos y clínicos del aborto en el Hospital "Gervasio Vera Custodio" de enero-diciembre 2013.

\section{MATERIALES Y METODOS}

Previo consentimiento del Departamento de Estadística del Hospital "Gervasio Vera Custodio", se incorporaron al estudio 207 historias clínicas con diagnóstico de aborto, las cuales incluyeron datos como: edad materna, edad gestacional, control prenatal, procedencia, realización de legrado y complicaciones posterior al mismo.

Se eligieron un total de 207 historias clínicas de mujeres con diagnóstico de aborto procedentes del servicio de ginecología del Hospital "Gervasio Vera Custodio" de Upata Estado Bolívar. Enero-Diciembre 2013. Previo consentimiento del Departamento de Estadística del Hospital "Gervasio Vera Custodio", se analizaron los siguientes datos: edad materna, edad gestacional, control prenatal, procedencia, realización de legrado y complicaciones posterior al mismo. Estos datos fueron procesados por medio del programa estadístico SPSS 20.0 español versión Windows. Se realizaron tablas de una y doble entrada según sea el caso. Se aplicó la prueba no paramétrica $\mathrm{chi}^{2}$ de Pearson para comparar los grupos de mujeres que se controlaron el embarazo vs las que no se lo controlaron, y de igual forma la edad gestacional y materna. Además se utilizó el programa Microsoft Excel 2010 para dar formato a las tablas. El valor de $\mathrm{p}<0,05$ fue considerado como estadísticamente significativo en todos los casos.

\section{RESULTADOS}

De 207 pacientes que abortaron fueron analizados 200 historias clínicas que conformaron 


\section{Pérez-Arciniegas et al}

la muestra; la edad materna promedio fue de $24 \pm 7$ años, siendo el grupo etario más frecuente el de 20 a 30, con una frecuencia de $42,0 \%(n=84)$ seguido de las pacientes de $12-20$ con $36,5 \%(n=73)$. La procedencia más frecuente fue Upata con 70,5\% $(\mathrm{n}=141)$ de los casos (Cuadro 1).

La edad gestacional promedio fue de $10 \pm 5$ semanas y el intervalo más frecuente fue de 1 a 8 semanas, representando 50,0\% $(n=100)$ de los casos registrados, $93,5 \%(\mathrm{n}=187)$ no tuvo control prenatal y el tipo de aborto más frecuente fue el espontaneo con 98,5\% ( $\mathrm{n}=197)$ (Cuadro 2).

En relación al manejo, el legrado uterino fue el más utilizado, en 97\% $(n=194)$ de los casos. $8,5 \%$ del total evaluado presentaron complicaciones, siendo la sepsis la más frecuentemente encontrada con $41,2 \%(n=7)$, seguido de la hemorragia transvaginal con $35,3 \%$ $(n=6)$ (Cuadro 3).

Las pacientes que cumplieron el control prenatal, 38,5\% $(\mathrm{n}=5)$ sen cuentran en los grupos comprendidos de 21 a 30 años y de 31 a 40 años; con menor frecuencia se visualiza $23,1 \%(n=3)$ en el grupo de 12 a 20 años. Por otro lado, las pacientes que no siguieron un control, 42,3\%

\section{Cuadro 1}

Aspectos epidemiológicos de pacientes que abortaron

\begin{tabular}{lcc}
\hline \multicolumn{1}{c}{ Edad } & Frecuencia & Porcentaje \\
\hline 12 y 20 & 73 & 36,5 \\
$21-30$ & 84 & 42,0 \\
$31-40$ & 7 & 3,5 \\
Total & 200 & 100,0 \\
Procedencia & & \\
Upata & 141 & 70,5 \\
El Palmar & 19 & 9,5 \\
El Manteco & 11 & 5,5 \\
Guasipati & 5 & 2,5 \\
Otras * & 24 & 12,0 \\
Total & 200 & 100,0 \\
\hline
\end{tabular}

*Otras: San Félix $(n=4)$, Kilómetro 88 ( $n=3)$, El Callao $(n=4)$, Santa Elena $(n=4)$, Ciudad Bolívar $(n=4)$, Tumeremo $(n=4)$, Táchira $(n=1)$ $(n=79)$ corresponden a edades comprendidas entre 21 a 30 años, seguido de un $37,4 \%(n=70)$ con edades comprendidas entre 12 a 20 años y con menos frecuencia, $16,6 \%(n=31)$ de 31 a 40 años. No se encontraron diferencias estadísticamente significativas al relacionar edad materna y control prenatal $(\mathrm{p}=0,21)$ (Cuadro 4).

$54,8 \%$ de las pacientes que abortaron tenían entre 12 y 20 años de edad y de 1 a 8 semanas de gestación, similar a lo observado en las pacientes con 21 a 30 años y la misma edad gestacional con $51,2 \%$; en las pacientes que abortaron con edad de 41 años y más la edad gestacional más frecuente fue la de 9-16 semanas con $42,9 \%$. El hallazgo de estos datos presntó diferencias estadísticas significativas $(p=0,02)$ (Cuadro 5).

\section{DISCUSIÓN}

La pérdida del embarazo durante el primer trimestre es la complicación más frecuente de la gestación, de modo que un porcentaje no

\section{Cuadro 2}

Frecuencia de edad gestacional, control prenatal y tipo de aborto

\begin{tabular}{lcc}
\hline $\begin{array}{c}\text { Edad } \\
\text { gestacional } \\
\text { (semanas) }\end{array}$ & Frecuencia & Porcentaje \\
\hline$<1$ & 2 & 1,0 \\
$1-8$ & 100 & 50,0 \\
$9-16$ & 76 & 38,0 \\
$17-22$ & 22 & 11,0 \\
Total & 200 & 100,0 \\
Control del & & \\
embarazo & 13 & 6,5 \\
Sí & 187 & 93,5 \\
No & 200 & 100,0 \\
Total & & \\
Tipo de & & \\
aborto & 197 & 98,5 \\
Espoontáneo & 3 & 1,5 \\
Provocado & 200 & 100,0 \\
Total & & \\
\hline
\end{tabular}

\section{Revista Biomédica}




\section{Epidemiología clínica del aborto en Venezuela}

Cuadro 3

Manejo y complicaciones de pacientes que abortaron

\begin{tabular}{lcc}
\hline $\begin{array}{c}\text { Legrado } \\
\text { uterino }\end{array}$ & Frecuencia & Porcentaje \\
\hline Sí & 194 & 97,0 \\
No & 6 & 3,0 \\
Total & 200 & 100,0 \\
Tipo de & & \\
complicación & & \\
Sepsis & 7 & 41,2 \\
Hemorragia & 6 & 35,3 \\
transvaginal & & 11,8 \\
Anemia & 2 & 11,8 \\
Otras* & 2 & 100,0 \\
Total & 217 & \\
\hline
\end{tabular}

* Hiperglicemia, hipertensión arterial

despreciable de mujeres sufrirán algún tipo de aborto a lo largo de su vida reproductiva (15).

En la presente investigación la edad materna en la que ser presentó mayor número de casos de aborto fue de 24 más o menos 7 años, similar a lo reportado por Tarqui-Mamani et al 2010 (25), cuya edad promedio reportada fue de 25 más o menos 6 años. En relación a los grupos etarios se encontró que el más frecuentemente afectado fue el de 20 a 30 con $42,0 \%$, a diferencia

\section{Cuadro 4 \\ Control prenatal en pacientes que abortaron}

\begin{tabular}{|c|c|c|c|c|c|c|}
\hline \multirow[t]{3}{*}{ Edad } & \multicolumn{4}{|c|}{ Control del embarazo } & \multicolumn{2}{|c|}{ Total } \\
\hline & \multicolumn{2}{|c|}{$\mathrm{Si}$} & \multicolumn{2}{|c|}{ No } & \multirow[b]{2}{*}{$\mathrm{N}$} & \multirow[b]{2}{*}{$\%$} \\
\hline & $\mathrm{N}$ & $\%$ & $\mathrm{~N}$ & $\%$ & & \\
\hline $12-20$ & 3 & 23,1 & 70 & 37,4 & 73 & 36,5 \\
\hline $21-30$ & 5 & 38,5 & 79 & 42,3 & 84 & 42,0 \\
\hline $31-40$ & 5 & 38,5 & 31 & 16,6 & 36 & 18,0 \\
\hline 41 y más & 0 & 0,0 & 7 & 3,7 & 7 & 3,5 \\
\hline Total & 13 & 100,0 & 187 & 100,0 & 200 & 100,0 \\
\hline
\end{tabular}

de lo reportado por Reyes-Aguilera (26), quien en su estudio realizado en 100 pacientes en el año 2009, encontró que el grupo más afectado fue el de 15 a 20, y similar a lo que reportó Farias-Noyola et al en 2010 (27) en el que fue el de 21-30 años de edad con 46,2\% de los casos; estas diferencias y semejanzas pueden deberse al contexto en el que se realizaron los trabajos, ya que el primero fue realizado en Cuba y el segundo en México.

La edad gestacional más frecuente fue de 1 a 8 semanas, representando $50,0 \%$ de los casos registrados, a diferencia de lo reportado por Farías-Noyola et al en 2010 (27) quien encontró que la edad gestacional fue de 9-12 semanas en

\section{Cuadro 5}

Edad gestacional de aborto, de acuerdo con la edad materna

\begin{tabular}{|c|c|c|c|c|c|c|c|c|c|c|}
\hline \multirow{3}{*}{$\begin{array}{c}\text { Edad } \\
\text { gestacional } \\
\text { (semanas) }\end{array}$} & \multicolumn{8}{|c|}{ Edad (años) } & \multicolumn{2}{|c|}{ Total } \\
\hline & \multicolumn{2}{|c|}{$12-20$} & \multicolumn{2}{|c|}{$21-30$} & \multicolumn{2}{|c|}{$31-40$} & \multicolumn{2}{|c|}{41 y más } & \multirow[b]{2}{*}{$\mathrm{N}$} & \multirow[b]{2}{*}{$\%$} \\
\hline & $\mathrm{N}$ & $\%$ & $\mathrm{~N}$ & $\%$ & $\mathrm{~N}$ & $\%$ & $\mathrm{~N}$ & $\%$ & & \\
\hline$<1$ & 1 & 1,4 & 0 & 0,0 & 0 & 0,0 & 1 & 14,3 & 2 & 1,0 \\
\hline $1-8$ & 40 & 54,8 & 43 & 51,2 & 15 & 41,7 & 2 & 28,6 & 100 & 50,0 \\
\hline $9-16$ & 22 & 30,1 & 35 & 41,7 & 16 & 44,4 & 3 & 42,9 & 76 & 38,0 \\
\hline $17-22$ & 10 & 13,7 & 6 & 7,1 & 5 & 13,9 & 1 & 14,3 & 22 & 11,0 \\
\hline Total & 73 & 100,0 & 84 & 100,0 & 36 & 100,0 & 100,07 & & 200 & 100,0 \\
\hline
\end{tabular}

$\mathrm{Chi}^{2}=19,04$; grados de libertad=9; valor de $\mathrm{p}=0,02$ 
Pérez-Arciniegas et al

$41 \%$ de los casos seguido de 1-8 semanas con $34,8 \%$ (15). No existió control prenatal en 93,5\%, cifra mucho más elevada al $15.1 \%$ reportado por Tarqui-Mamani et al en 2010, este es un dato alarmante debido a que el control prenatal constituye una herramienta fundamental para detectar problemas en el embarazo y, así, elegir el posible tratamiento a seguir (28).

En la presente investigación, se encontró que la mayoría de las pacientes que abortaron eran jóvenes y no llevaron un control médico del embarazo, lo que pudo haberse debido al desconocimiento sobre la prevención de eventualidades como el aborto. Por lo anterior, se debe seguir insistiendo y realizar charlas sobre este tema para evitar, o al menos disminuir estas cifras de aborto que constituye un problema de salud pública (28).

La edad gestacional más frecuente fue de 1 a 8 semanas, siendo las pacientes de 12 a 20 años las más afectadas con 54,8\% de los casos. En un estudio anterior, se registró que el índice entre los casos de aborto durante el primer trimestre observados y esperados, era mayor en las que tenían menos de 18 años y menor en las de 20 a 35 años, con un ascenso abrupto después de esa edad (29). Lo anterior es similar a lo encontrado en este estudio en el que las de menos edad fueron las que más frecuentemente abortaron.

En relación al manejo, el legrado uterino fue el más común con 97\%; de estos casos, 8,5\% presentaron complicaciones, en los que la sepsis fue la más frecuente con $41,2 \%$, seguido de la hemorragia transvaginal con $35,3 \%$. Es de resaltar que el legrado uterino puede ser obstétrico o ginecológico; en la presente investigación, fue utilizado el obstétrico que generalmente está indicado en casos de aborto incompleto, y las complicaciones más frecuentemente reportadas son las infecciones post-legrado y la perforación uterina, que es una causa de hemorragia transvaginal (30).

En conclusión, se encontró una alta frecuencia de pacientes que abortaron sin haber llevado un control adecuado del embarazo; el grupo etario más afectado fue el de 21-30 y la edad gestacional más frecuentemente afectada fue de 1 a 8 semanas. Debido a estos hallazgos se recomienda realizar campañas de concientización a la población en general, haciendo hincapié de la importancia que tiene el control del embarazo en la prevención o al menos la disminución de las cifras de este problema de salud pública y realizar otros trabajos con la finalidad de determinar las causas específicas de aborto en esta comunidad.

\section{REFERENCIAS}

1. Kahun Medical Papyrus, Manuscript for the health of mother and child translation by Stephen Quirke University College London, 2002.

2. Palés M, Villar C. Diccionario de la lengua española. España: Espasa Calpe, S.A; 2001.

3. Hijona J. Factores asociados al aborto espontáneo. [tesis doctoral]. Granada. Universidad de Granada.2009.

4. Ganatra B, Tunçalp Ö, Bart Johnston H, Johnson B, Metin Gülmezoglu A, Temmerman From concept to measurement: operationalizing WHO's definition of unsafe abortion. Boletín de la Organización Mundial de la Salud 2014;92:155. Disponible en: http://dx.doi. org/10.2471/BLT.14.136333.

5. Anuario de Morbilidad. MPPS. Venezuela. 2009. Disponible en: http://www.mpps.gob.ve/index. php?option $=$ com_phocadownload\&view $=$ category \& $\mathrm{id}=41$ :anuariosestadsticos\&Itemid $=915$

6. Código penal de Venezuela. Artículos 17, 432 y 433. Disponible en: http://www.defiendete.org/ $\mathrm{html} /$ denteres/LEYES\%20DE\%20VENEZUELA/ LEYES\%2... 10/14/2011)

7. Ruiz Parra A. Aborto. En: Guías para el manejo de urgencias. Cap X. Colombia. Departamento de Ginecología y Obstetricia. Universidad Nacional de Colombia. 2013. p 906-911.

8. Menéndez-Velázquez J. Aspectos médicos. El manejo del aborto espontáneo y de sus complicaciones. Gac Méd Méx Vol.139, Suplemento No. 1, 2003.

9. Neira Miranda, Jorge. 2002. Aborto. aspectos clínicos y epidemiológicos . [en línea] Ars Médica. Revista de Estudios Médico Humanísticos. Vol. $6 \mathrm{~N}^{\circ} 6$. Pontificia Universidad Católica de Chile.

10. Villela Cortés F, Linares Salgado J. Diagnóstico genético prenatal y aborto. Dos cuestiones de

\section{Revista Biomédica}




\section{Epidemiología clínica del aborto en Venezuela}

eugenesia y discriminación. Revista de Bioética y Derecho. 2012; 24: 31-43.

11. Zapardiel Gutiérrez I, De la fuente Valero J, Bajo Arenas J.M. Guía práctica en urgencias en obstetricia y ginecología. (acorde a los protocolos de la S.E.G.O.). $1^{\circ}$ ed. Madrid: Nabe; 2008.

12. Casasco G, Di Pietrantonio E. Aborto: guía de atención. Revista del Hospital Materno Infantil Ramón Sardá. 2008; 27:33-41.

13. González Rivera A. Amenaza de Aborto. Revista médica de costa rica y Centroamérica. 2011; 599:495498

14. Faneite $P$, Amato $R$, Rodríguez F, Faneite J, Rodríguez Y, Rivera C. Aborto séptico en el Hospital "Dr. Adolfo Prince Lara" 1977-2006. Rev Obstet Ginecol Venez. 2007;67(3):174-178

15. Romero Guadix B, Martínez Navarro l, González Paredes A, Fontes Jiménez J. Epidemiología del aborto de repetición. Prog Obstet Ginecol. 2012;55(7):312-320

16. Bolívar Mejía A, Calvo Betancourt L, Avilán S, Contreras García G. Aborto recurrente de etiología autoinmune. Rev. Méd. Risaralda 2013; 19 (1):8185

17. Pacheco J, De Michelena I, Orihuela P. Enfoque actual del aborto recurrente. An Fac med. 2009;70(2):123-34

18. Méndez Vásquez J. Aspectos Médicos: el manejo del aborto espontaneo y sus complicaciones. Gac Méd Méx. 2003; 139 ( Supl 1): 46-53

19. Alarcón Nivia M. Guía de manejo El legrado uterino, indicaciones, beneficios y riesgos. Revista de los estudiantes de medicina de la universidad industrial de Santander. 2007; 20:151-156

20. Schiavon R. Aborto médico: Alternativas terapéuticas actuales. Gac Méd Méx. 2003; 139 (Supl 1):54-63

21. Donoso E, Oyarzún E. Análisis comparativo de la mortalidad materna en chile, cuba y estados unidos de Norteamérica. Rev Chil Obstet GINECOL 2004;
69(1): 14-18

22. Doblado I, De la Rosa I, Junco A. Aborto en la adolescencia un problema de salud. Rev Cub de Obst y Ginecol 2010; 36(3)409-421

23. L.J. Escobar y Álvaro. Complicaciones médicas del aborto. Disponible en: http://es.scribd.com/ doc/133905811/L-J-Escobar-Complicacionesmedicas-del-aborto-Servicio-de-Urgenciasdel-Hospital-Rafael-Mendez-Lorca-MurciaEspana\#scribd

24. Fernández Cantón S, Gutiérrez Trujillo G, Viguri Uribe R. Estadísticas vitales. Bol Med Hosp Infant Mex 2012;69(1):77-80.

25. Tarqui-Mamani C, Barreda A, Barreda $M$, Sanabria-Rojas H. Prevalencia del intento de interrumpir el embarazo y factores asociados en una comunidad urbano marginal de Lima, Perú, 2006. Rev Peru Med Exp Salud Publica. 2010; 27(1): 38-44.

26. Reyes-Aguilera $M$, Merencio-Leyva N, RodríguezLlano JR, Soria-Pérez S. Aspectos clínicos y epidemiológicos del aborto inducido en pacientes del área de salud de Mayarí. Correo Científico Médico de Holguín. 2011;15(3). [En línea] disponible en: http://www.cocmed.sld.cu/no153/pdf/resut04.pdf [citado, 10 de agosto, 2015].

27. Farias-Noyola JG, Carbajal-Ruíz FA. Aspectos Epidemiológicos y Clínicos del Aborto en el Benemerito Hospital General Juan María de Salvatierra de La Paz, Baja California Sur en el Periodo de 20072008. Bol Clin Hosp Infant Edo Son 2010 mayo; 27(2): 119-121.

28. Organización Mundial de la Salud. Nuevo modelo de control prenatal de la OMS. Ginebra, Suiza. 2003.

29. Reece EA, Hobbins JC, Gant NF. Obstetricia Clínica. $3^{\mathrm{a}}$ edición. Madrid. Editorial Médica Panamericana. 2010.

30. Alarcón-Nivia MA. El legrado uterino, indicaciones, beneficios y riesgos. MÉDICAS UIS 2008;20:151-56. 